\title{
Numerical analysis of MHD Casson boundary layer nanofluid flow over porous stretching surface with the effects of radiation and chemical reaction
}

\author{
Veera Reddy Karnati ${ }^{1}$, Venkata Ramana Reddy GURRAMPATI ${ }^{2}$, Seethamahalakshmi \\ VYAKARANAM ${ }^{3}$, and Y Hari Krishna ${ }^{4}$
}

${ }^{1}$ Reearch cholar Department of Mathematics, Koneru Lakshmaiah Education Foundation, Vaddeswaram-522502.

${ }^{2}$ Department of Mathematics, Koneru Lakshmaiah Education Foundation, Vaddeswaram-522502.

${ }^{3}$ Department of Mathematics, PVP Siddartha Institue of Technology, Kanur,India-520007.

${ }^{4}$ Department of Mathematics, ANURAG Engineering College, Anathgiri (v), Kodad, Suryapet, Telangana, India-508206

\begin{abstract}
In this article numerical analysis of incompressible, two dimensional and mixed convective MHD Casson fluid flows over a stretching surface with porosity under the influence of radiation and chemical reaction as well as viscous dissipation considered. By utilizing suitable similarity analysis, the governing PDEs (Partial differential equations) with its respective boundary conditions were transformed to dimensionless forms. The resulting ODEs (Ordinary differential equations) along with corresponding boundary conditions were solved via shooting technique combined with Runge-kutta-Fehlberg method. The outcomes of this study illuminates that velocity, temperature and concentration fields decreases due to the thickness of the boundary layer as we go away from the stretching sheet surface and falling of velocity observed in Casson fluid parameter. Under some restriction the resultant outcome were compared with previous published results and is found in admirable agreement.
\end{abstract}

\section{INTRODUCTION}

Depends on viscosity the ratio of shear stress with the rate of strain inside the fluid can be categorized as linear, nonlinear or plastic. The shear stress is proportional linear to the rate of strain, in Newtonian fluid, hear the proportionality constant is the dynamic viscosity coefficient. While in case of non-Newtonian the shear stress is nonlinearly proportional to the rate of strain, here the constant coefficient doesn't defined as even it is reliant on time. Further, plastic deformation retains if shear stress not depends on rate of strain. When undergoing deformation the material exhibit both combine characteristics of viscous and elastic property is known as viscoelastic. The viscoelastic property disappears with the rate of shear, time and temperature.

In fluid dynamics, dissipation is the energy conversion from one form to another. For example if fluid flow needs kinetic energy i.e. velocity of the fluid changes energy in to internal energy by taking it from the motion. The kinetic energy is dissipated. The rise of the fluid velocity outcomes the rise up the temperature of the fluid.
Depending upon the Eckert number (nondimensional quantity) viscous dissipation varies. Viscous dissipation is applied in geophysical flows and industries. Santoshiet al. [1] discussed the effects of chemical reaction on boundary layer flow upon a stretching sheet along with viscous dissipation. Dash et al. [2] explored the impact of heat and mass transfer on boundary layer fluid flow past through a moving vertical porous plate along with magnetic field in the transverse direction in the presence of heat source and chemical reaction. Tiegang et al. [3] researched an unsteady incompressible, boundary layer flow of stagnation-point with mass transfer. Zigta [4] analyzed the effect of unsteady, incompressible, magneto hydrodynamics filled with viscoelastic fluid in an infinite vertical porous channel inserted in a porous medium. Numerous investigators talked over different traits of stretching flow problem [5]. Reddy et al. [6] studied an unsteady MHD free flow of a Casson fluid past an oscillating vertical plate with constant wall temperature. Reddy et al. [7] analyzed the impact of radiation and heat generation on MHD boundary layer fluid flow over a stretching porous surface through porous medium with mass 
transfer. Raja Sekhar et al. [8] studied an unsteady oscillatory MHD slip fluid flow over a planer channel with the impact of chemical reaction and heat source. Chiam [9] discussed the boundary layer flow on stretching plate with distribution of velocity by power law in the presence of magnetic field in transverse direction. Elbashbeshy et al. [10] developed Maxwell nanofluid flow with heat and mass transfer over a stretching surface inserted in a porous medium in the existence of a heat source. Suresh et al. [11] analyses the impact of thermal radiation on free convective MHD fluid flow over a vertical porous moving plate in presence of chemical reaction. Liao et al. [12] explained comprehensive account of the boundary layers through a vertical plate inserted in a porous medium. Reddy et al. [13] discussed the impact of heat radiation on boundary layer MHD nanofluid slip velocity flow, over a stretching surface with boundary conditions. Shateyi et al. [14] explained the free convection of combined heat and mass transfer effects toward an unsteady stretching porous sheet along with radiation, viscous dissipation and chemical reaction. Chamka et al. [15] studied thermophoretic MHD heat and mass transfer stream above plane surface. Gebhart et al. [16] discussed some general characteristics of similarity circumstances for flow over in convection plumes and surfaces. Vajravelu et al. [17] considered the phenomenon of heat transfer and flow through a nonlinearity enlarging sheet.
Rafael [18] considered transfer of heat and fluid flow in a stretching surface through a porous medium. Khan et al. [19] examined boundary layer flow of viscoelastic and transfer of heat over continuous exponential stretching sheet. Akyildiz et al. [20] developed the viscoelastic fluid flow with mass transfer of species which are chemically reactive over an extending sheet. Pop et al. [21] developed the steady boundary layer nanofluid flow with heat transfer over a widening surface. Aziz et al. [22] investigated the effect of heat transfer on boundary layer nanofluid flow with convective boundary condition over an enlarging surface.

The main objective of the present study is to analyze the effect of MHD Casson boundary layer nanofluid flow upon a stretching porous sheet in the existence of radiation, chemical reaction along with viscous dissipation. The governing PDEs transformed to ODEs then the resulting ODEs are solved by shooting technique along with Rungekutta-Fehlberg method.

\section{Mathematical formulation}

Consider the numerical investigation on an incompressible, steady, two-dimensional, laminar, viscous boundary layer flow of an electrically conducting MHD Casson fluid towards a stretching surface with chemically reactive species experiencing chemical reaction.

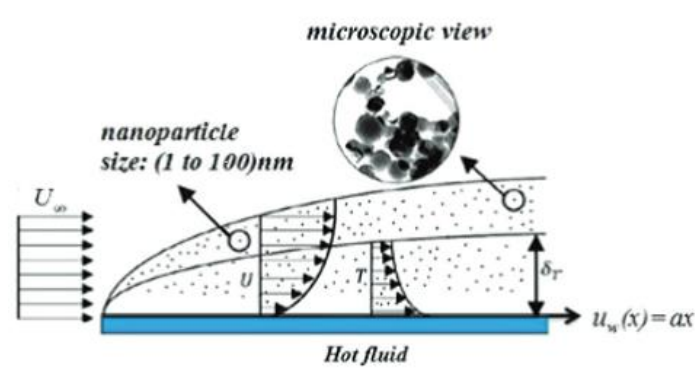

Fig.1. Geometry of the flow under consideration

The plate has been taken in $\mathrm{x}$-direction along with stretched velocity $u_{w}(x)=a x,(a>0)$ as shown in Fig.1. Further, the magnetic field applied in $y$ direction to the fluid flow. At the surface $T_{f}, u_{w}$,
$C_{w}$ represent fluid temperature, velocity and concentration of nanoparticle respectively. 
The state of Casson fluid rheological equation is given by

$$
\tau_{i j}=\left\{\begin{array}{l}
2\left(\frac{\mu_{B}+P_{y^{\prime}}}{\sqrt{2 \pi}}\right) e_{i j}, \pi>\pi_{c,} \\
2\left(\frac{\mu_{B}+P_{y^{\prime}}}{\sqrt{2 \pi_{c}}}\right) e_{i j}, \pi<\pi_{c,}
\end{array}\right.
$$

where $\pi=e_{i j} e_{i j}$ and $e_{i j}$ represents the component of $(\mathrm{i}, \mathrm{j})^{\text {th }}$ distortion rate, $\pi$ being the resulted from distortion rate itself, the product $\pi_{c}$ is a critical value reliant over non-Newtonian form, $\mu_{B}$ represents the non-Newtonian fluid viscosity relevant to a fluid of plastic dynamic and $P_{y}$ gives the fluid yield pressure. The governing conservation equations under the boundary layer approximation, are as follows:

$\frac{\partial u}{\partial x}+\frac{\partial v}{\partial y}=0$ $u \frac{\partial u}{\partial x}+v \frac{\partial u}{\partial y}=-\frac{1}{\rho_{f}} \frac{\partial p}{\partial x}-\frac{\sigma B_{0}^{2}(x)}{\rho_{f}}(u)-\frac{v}{K^{*}} u+v\left(1+\frac{1}{\beta}\right)\left(\frac{\partial^{2} u}{\partial x^{2}}+\frac{\partial^{2} u}{\partial y^{2}}\right) \begin{aligned} & \text { Here } \sigma^{*} \text { represents the constant of Stefan- } \\ & \text { Boltzmann, } k \text { indicates thermal conductivity and }\end{aligned}$

$u \frac{\partial v}{\partial x}+v \frac{\partial v}{\partial y}=-\frac{1}{\rho_{f}} \frac{\partial p}{\partial y}-\frac{\sigma B_{0}^{2}(x)}{\rho_{f}}(v)-\frac{v}{K^{*}} u+v\left(1+\frac{1}{\beta}\right)\left(\frac{\partial^{2} u}{\partial x^{2}}+\frac{\partial^{2} u}{\partial y^{2}}\right)$ $k^{*}$ gives the coefficient of mean absorption.

We use similarity transformation to solve equations (2), (3), (4), (5), (6), (7) and (8)

$u \frac{\partial T}{\partial x}+v \frac{\partial T}{\partial y}=\frac{\mu}{C_{p}}\left(\frac{\partial u}{\partial y}\right)^{2}+\alpha \frac{\partial^{2} T}{\partial y^{2}}+\phi\left[D_{B} \frac{\partial C}{\partial y} \frac{\partial T}{\partial y}+\frac{D_{T}}{T_{\infty}}\left(\frac{\partial T}{\partial y}\right)^{2}\right]-\frac{\alpha}{\rho_{f}}\left(\frac{\partial q_{r}}{\partial y}\right)$ $\psi=\sqrt{a v} x f(\eta), \theta(\eta)=\frac{T-T_{m}}{T_{\infty}-T_{m}}, \beta(\eta)=\frac{C-C_{\infty}}{C_{\omega}-C_{\infty}}, \eta=y \sqrt{\frac{a}{v}}$

$u \frac{\partial C}{\partial x}+v \frac{\partial C}{\partial y}=D_{B} \frac{\partial^{2} C}{\partial y^{2}}+\frac{D_{T}}{T_{\infty}^{\prime}} \frac{\partial^{2} C}{\partial y^{2}}-k\left(C-C_{\infty}\right)$

The stream function velocity can be defined as

$$
u=\frac{\partial \psi}{\partial y}, v=-\frac{\partial \psi}{\partial x}
$$

Here $u$ and $v$ are the velocity components in the direction of $x$ and $y$ respectively, $p$ - the fluid pressure, $\rho_{f}{ }^{-}$the base fluid density, $\rho_{p}$ particles density, $v$ - kinematic viscosity of the www.psychologyandeducation.net

base fluid, $\sigma-$ the electrical conductivity, $\rho$ fluid density, $T$ - temperature, $\alpha$ - thermal diffusivity, $\tau=\frac{(\rho \mathrm{c})_{p}}{(\rho c)_{f}}$ is the heat capacity ratio of nanofluid and the base fluid, $D_{B}, D_{T}$ are the coefficients of Brownian and thermophoretic diffusion, $\mu$ - dynamic viscosity, $C_{p}$ - specific heat.

The boundary conditions for Equations (2), (3), (4), (5) and (6) are given as

$$
\begin{aligned}
& u=u_{w}+L \frac{\partial u}{\partial y}, v=v_{w},-k \frac{\partial T}{\partial y}=h\left(T_{f}-T\right), C=C_{w} \text { at } y=0 \\
& u=0, v=0, T \rightarrow T_{\infty}, C \rightarrow C_{\infty} \text { as } y \rightarrow \infty
\end{aligned}
$$

Using Rosseland approximation equation (4) can be expressed as

$$
u \frac{\partial T}{\partial x}+v \frac{\partial T}{\partial y}=\frac{\mu}{C_{p}}\left(\frac{\partial u}{\partial y}\right)^{2}+\alpha \frac{\partial^{2} T}{\partial y^{2}}+\phi\left[D_{B} \frac{\partial C}{\partial y} \frac{\partial T}{\partial y}+\frac{D_{T}}{T_{\infty}}\left(\frac{\partial T}{\partial y}\right)^{2}\right]+\frac{\alpha}{\rho_{f}} \frac{16}{3}
$$


$u=a x f^{\prime}(\eta), u=-\sqrt{a v} f(\eta)$

here prime indicates differentiation w.r.to $\eta$.

Using equation (9) in equation (1) that will be satisfied, using equations (8)-(10) in equations (2)-(4), we will get the following ODEs.

$\left(1+\frac{1}{\beta}\right) f^{\prime \prime \prime}+f f^{\prime \prime}-\left(f^{\prime}\right)^{2}-\left(M+\frac{1}{K}\right) f^{\prime}=0$

$\left(1+\frac{4}{3} R\right) \theta^{\prime \prime}+\operatorname{Pr}\left[f \theta^{\prime}+N b \theta^{\prime} \beta^{\prime}+N t\left(\theta^{\prime}\right)^{2}+E c\left(f^{\prime \prime}\right)^{2}\right]=0$

$\beta^{\prime \prime}+\operatorname{Lef} \beta^{\prime}+\frac{N t}{N b} \theta^{\prime \prime}-\operatorname{Le\gamma } \beta=0$ $\begin{array}{lr}\text { with the boundary conditions } & \operatorname{Re}_{x}=\frac{x u_{w}(x)}{v} \\ f(0)=S, f^{\prime}(0)=1+A f^{\prime \prime}(0), \theta^{\prime}(0)=-B i(1-\theta(0))=0, \beta(0)=1 \text { at } \eta^{\nu}=0\end{array}$ $f^{\prime}(\infty) \rightarrow 0, \theta(\infty) \rightarrow 0, \beta(\infty) \rightarrow 0$ as $\eta \rightarrow \infty$

The dimensionless constants $\mathrm{M}, \mathrm{S}, \mathrm{R}, \mathrm{A}, \mathrm{Pr}, \mathrm{Nb}$, $\mathrm{Nt}, \mathrm{Ec}, \gamma, \mathrm{Le}, \mathrm{Bi}, \mathrm{K}$ represent the parameters of magnetic, suction, radiation, velocity slip, Prandtl number, Brownian motion, thermophoresis, Eckert number, Lewis number, chemical rate, Biot number and permeability respectively, which can be defined as

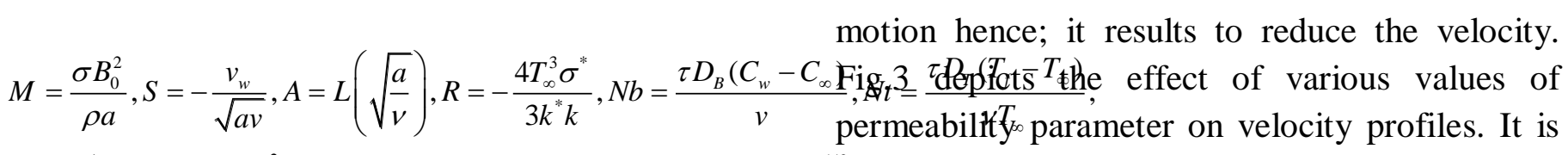
$K=\frac{K^{*} a}{v}, E c=\frac{u_{w}^{2}}{\rho_{f}\left(T_{f}-T_{\infty}\right)}, \gamma=\frac{k_{0} u\left(C_{w}-C_{\infty}\right)}{v}, \operatorname{Pr}=\frac{v}{\alpha}, B i=\frac{h(v / a)^{1 / 2} \text { bserved }}{k}$, LermedBility parameter, the velocity raises due to velocity profiles are shown in Fig.4. It is noticed porous medium increases the resistance to the flow which cases the fluid to decrease. The influence of the Casson fluid parameter $(\beta)$ on the

The Nusselt number $N u_{x}$, the skin friction coefficient $C_{f}$, and the Sherwood number $S h_{x}$, can be expressed as:

$$
N u_{x}=\frac{x q_{w}}{k\left(T_{w}-T_{\infty}\right)}, C_{f}=\frac{\tau_{w}}{\rho u_{w}^{2}} \text {, and } S h_{x}=\frac{x q_{m}}{D_{B}\left(C_{w}-C_{\infty}\right)}
$$

Where, $\tau_{w}$ is the surface shear stress, $q_{m}$ is the wall surface mass flux, and $q_{w}$ is the heat flux at the wall surface, given by:

$q_{w}=-\left(\frac{\partial T}{\partial y}\right)_{y=0}, q_{m}=-D_{B}\left(\frac{\partial C}{\partial y}\right)_{y=0}$

Using the dimensionless variables, we get $\frac{N u_{x}}{\sqrt{R_{x}}}-1+R \theta^{\prime}(0), \frac{S h_{x}}{\sqrt{R_{x}}}=-\beta^{\prime}(0), C_{f} \sqrt{R_{x}}=-f^{\prime \prime}(0)$

where $R e_{x}$ indicates the Reynolds number and is stated as:

Fig.2depicts the impact of the magnetic parameter (M), on velocity profiles. For both the cases in suction and injection, it is clear that for rising values of magnetic field parameter $(\mathrm{M})$, the velocity profiles decreases. This is because of the fact that the influence of magnetic field on electrically conducting fluid generates a drag force which cultivates the body force known as Lorentz force. This force diminishes the fluid (n) (n) (n) 
that for the raising values of Casson fluid parameter $(\beta)$ the fluid velocity decreases, the reason is that the fluid become more viscous with the growth of Casson fluid parameter $(\beta)$, therefore more resistance is offered, which reduces the boundary layer thickness. Fig. 5 depicts the temperature profile for different values of radiation parameter $(\mathrm{R})$. It is observed that the temperature enhances with an increasing values of radiation parameter. Because of the reason, for the raising surface heat flux leads to temperature grow up.

The variation of Prandtl number w.r.to the temperature profiles as shown in Fig.6. As Prandtl number $\mathrm{Pr}$ is in inversely related with thermal conductivity. So that for an enhancing value of Prandtl number, the capability of material to conduct heat falls so, the temperature of the fluid diminutions. Fig.7. represents the influence of slip parameter (A) on the velocity profiles. The slip parameter growths, the velocity decreases due fluid flow boundary layer thickness. Fig. 8 shows the variations in velocity w.r.to suction parameter (S). It is noted that the suction parameter rises, velocity falls. As the fluid particles tense into the wall with the increase of suction parameter hence the boundary layer reduces. Figs. (9) and (10) reveal the influence of thermophoresis parameter (Nt) on the distribution of temperature and concentration profiles respectively. It is identified that the temperature upturns with the growing the thermophoresis parameter. The heightening of thermophoretic effects outcomes the nanoparticles migration from the hot to the cold surface ambient fluid as a result of this the temperature rises in the boundary layer. Also it is noticed that the concentration drops with an growing thermophoresis parameter. Figs. (11) and (12) prepared to show the Brownian motion parameter $(\mathrm{Nb})$ impacts on the temperature and concentration profiles respectively. It is observed that both the temperature and concentration increases with an increasing the Brownian motion due to the thickness of thermal boundary layer. Fig. 13 represents the influence of Eckert number on the temperature distribution and it is visualized here to increase the Eckert number increases, temperature increases. Due to friction, the Eckert number values accelerate with the heat energy, which consequences in the enrichment of the temperature profile. Fig.14 depicts the concentration profiles with the impact of Lewis number. It is observed that the concentration decreases with an enhancing Lewis parameter. This is due to the ratio of diffusivity of momentum to Brownian diffusion. The influence of chemical reaction on concentration profiles discoursed with the assistance of Fig. 15. It can be identified that for an enhancing chemical reaction, the concentration profiles are decreases depletion in concentration. The impact of Biot number on the temperature profiles as shown in Fig.16. As Biot number evaluates the heat transfer resistance quotient from outside and on the surface, which causes the temperature profile increases.

For the validity of our results, the skin friction factor, the Nusselt and Sherwood number have been compared with those already published in literature as shown in Tables I and II. From tables it can be seen that the results achieved by the present code are found convincingly much closed to the published results [1].

Table III report the alterations in skin-friction, Nusselt and Sherwood factors against physical parameters, namely, M, K, B, R, Pr, A, S, Nt, Nb, Ec, Le and Bi respectively. Skin friction, Nusselt and Sherwood numbers are upturns when raising of the parameters M, K, B. Nusselt and Sherwood wood numbers are increases when enhancing all parameter but skin friction is constant when increasing of $\mathrm{R}, \mathrm{Pr}, \mathrm{Nt}, \mathrm{Nb}, \mathrm{Ec}$, Le and $\mathrm{Bi}$. 


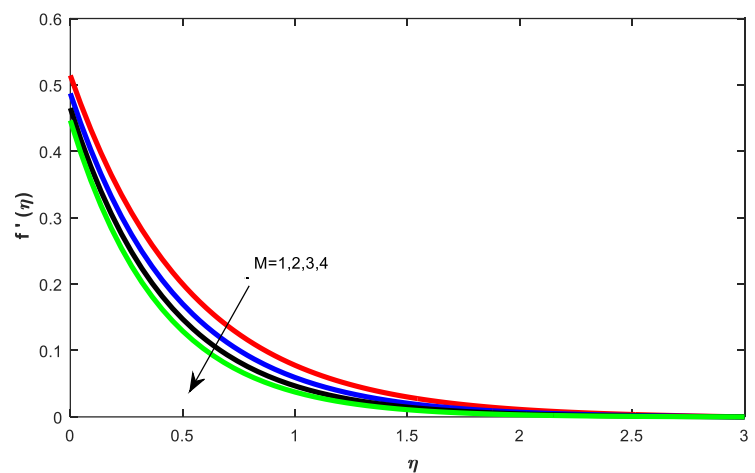

Fig.2.Velocity profiles for various values of magnetic parameter (M).

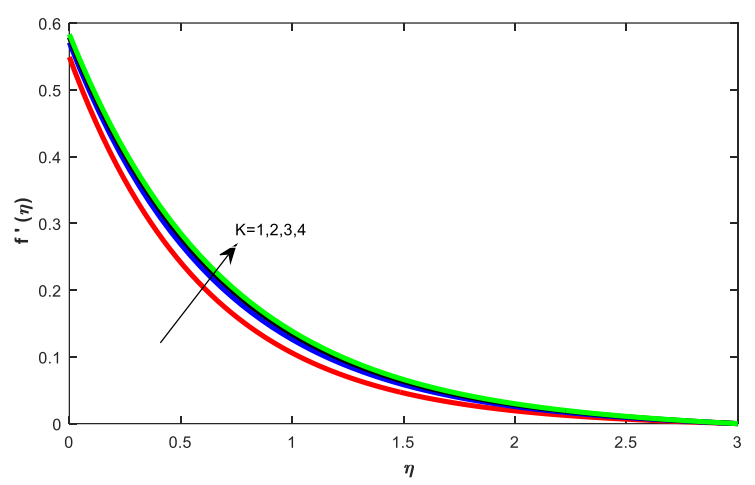

Fig.3.Velocity profiles for dissimilar values of permeability parameter $(\mathrm{K})$.

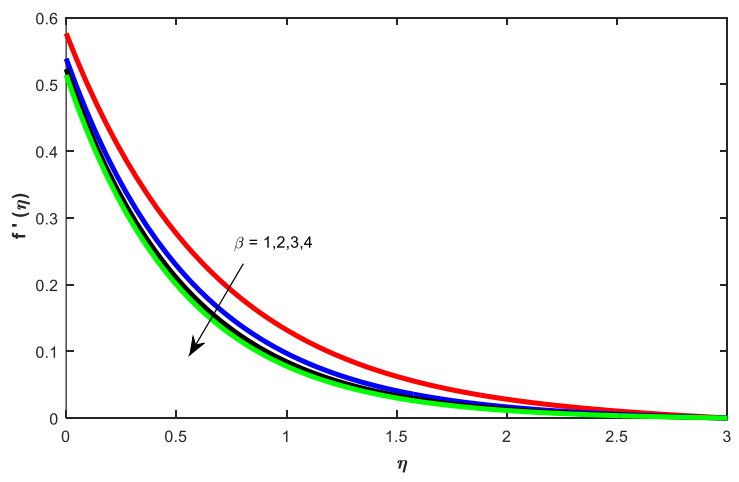

Fig.4. Velocity profiles for various values of Casson parameter $(\beta)$.

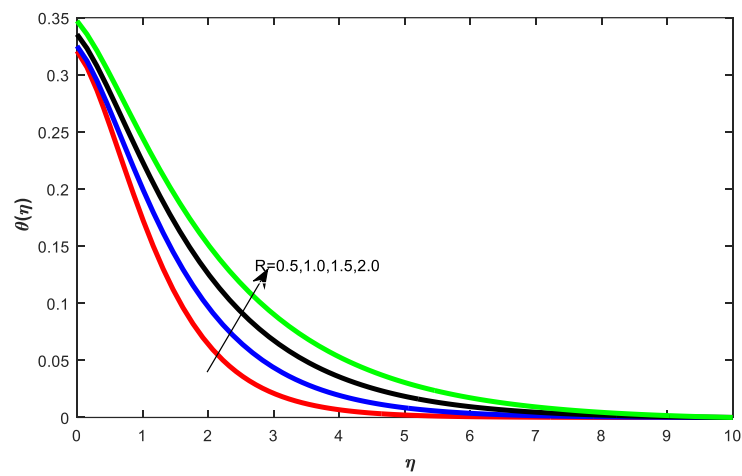

Fig.5.Temperature profiles for dissimilar values of radiation parameter $(R)$.

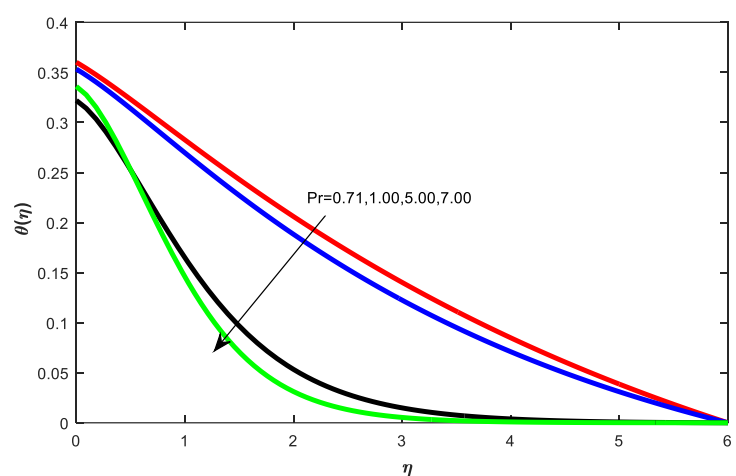

Fig.6.Temperature profiles for various values of Prandtl number (Pr).

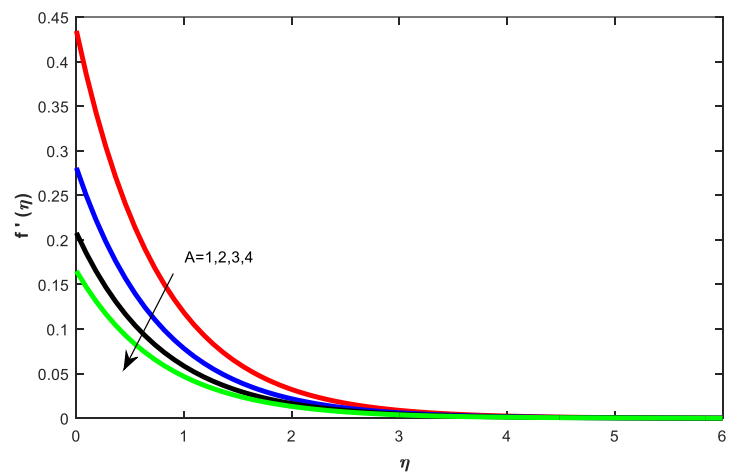

Fig.7.Velocity profiles for dissimilar values of slip parameter (A). 


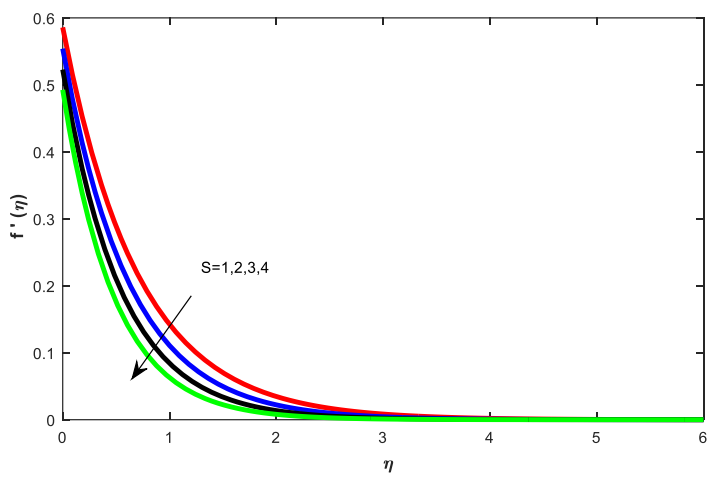

Fig.8.Velocity profiles for various values of suction parameter $(\mathrm{S})$.

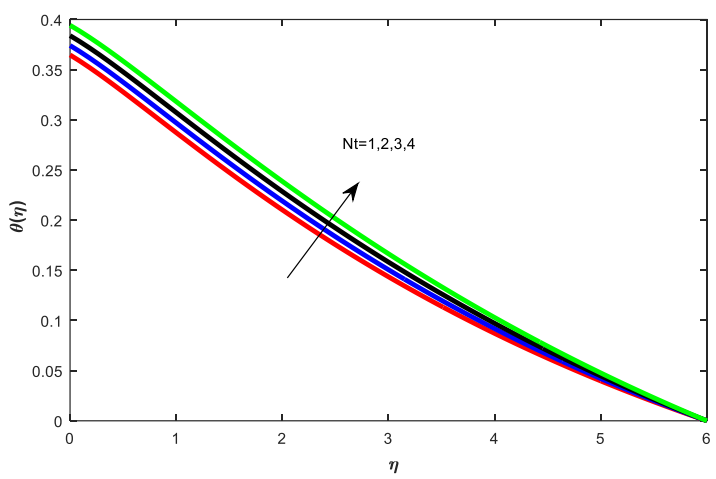

Fig.9.Temperature profiles for different values of thermophoresis parameter $(\mathrm{Nt})$.

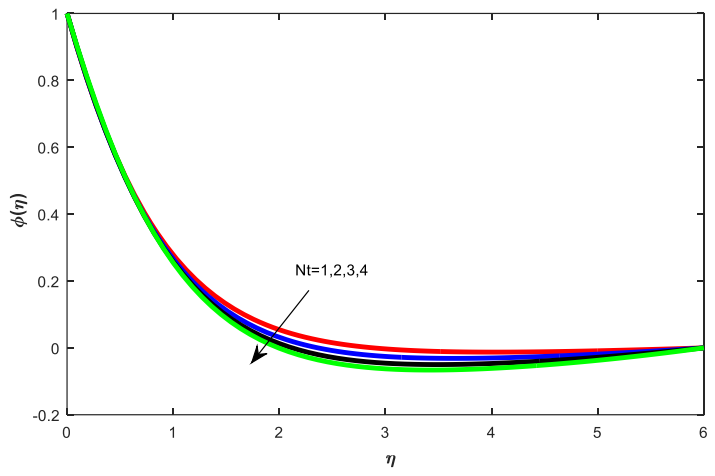

Fig.10.Concentration profiles for dissimilar values of thermophoresis parameter $(\mathrm{Nt})$.

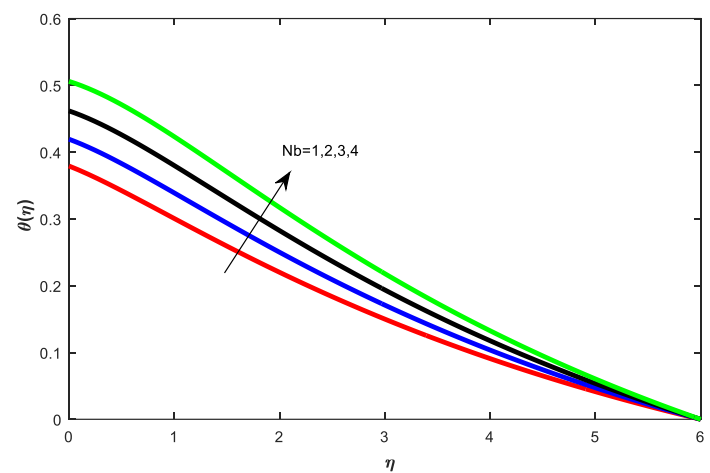

Fig.11.Temperature profiles for various values of Brownian motion parameter $(\mathrm{Nb})$.

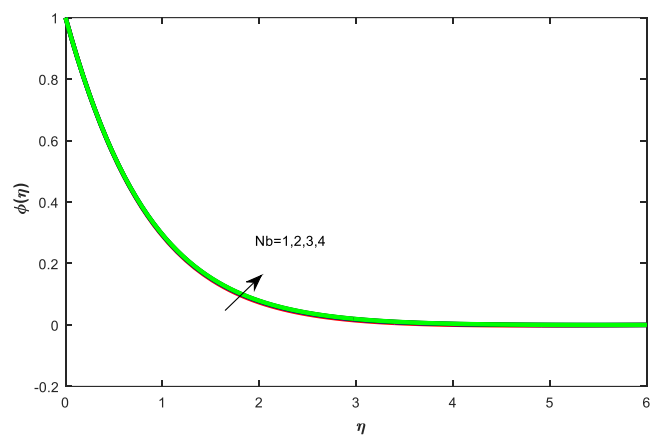

Fig.12.Concentration profiles for dissimilar values of Brownian motion parameter $(\mathrm{Nb})$.

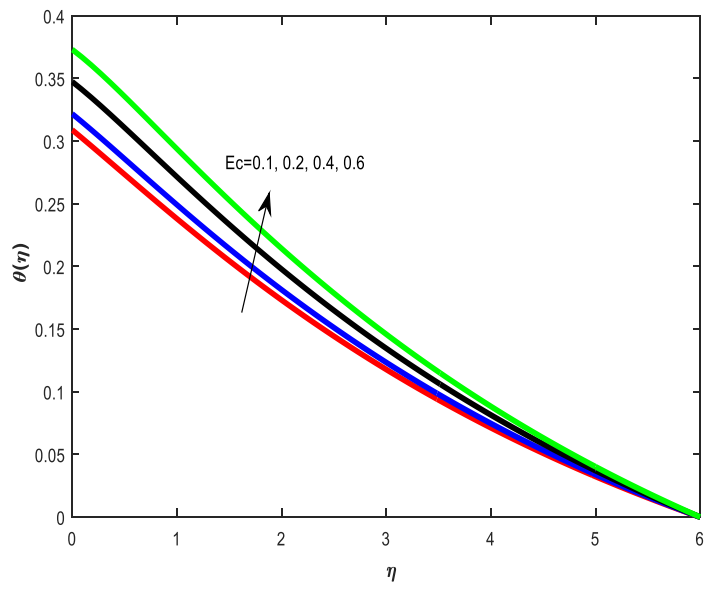

Fig.13.Temperature profiles for various values of Eckert number (Ec). 


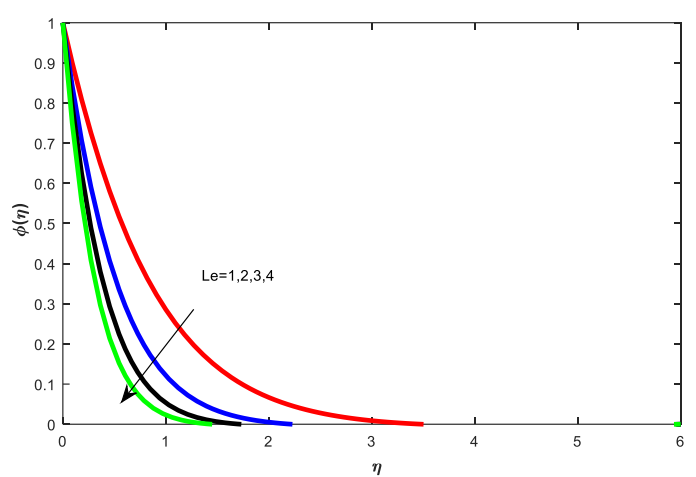

Fig.14.Concentration profiles for dissimilar values of Lewis number (Le).

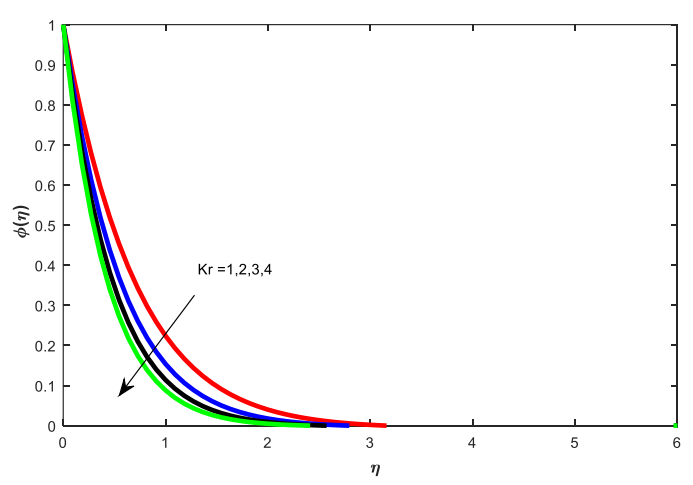

Fig.15.Concentration profiles for various values of chemical reaction parameter $(\mathrm{Kr})$.

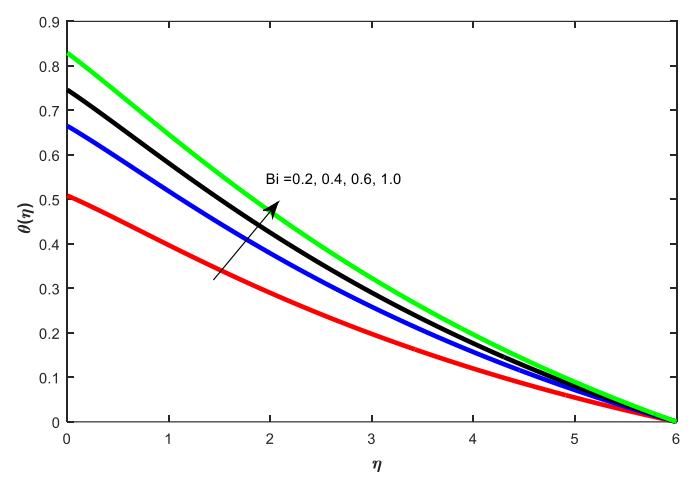

Fig.16.Temperature profiles for different values of Biot number $(\mathrm{Bi})$.

Table I. Comparative outcomes of skin friction coefficient $-f^{\prime \prime}(0)$ for various values of $M, S$ and A

\begin{tabular}{|l|l|l|l|l|}
\hline $\mathrm{M}$ & $\mathrm{S}$ & $\mathrm{A}$ & $\begin{array}{l}f^{\prime \prime}(0) \\
\text { Santoshi } \\
\text { Misra [1] }\end{array}$ & Present value \\
\hline 0.5 & 0.2 & 0.1 & 1.136756 & 1.136656 \\
\hline 1.0 & 0.2 & 0.1 & 1.282955 & 1.283954 \\
\hline 0.5 & 0.5 & 0.1 & 1.265184 & 1.268401 \\
\hline 0.5 & 0.2 & 0.5 & 0.8066351 & 0.8064753 \\
\hline
\end{tabular}

Table II. Comparative outcomes of Nusselt $\mathrm{r}-\theta^{\prime}(0)$ and Sherwood $-\beta^{\prime}(0)$ numbers

\begin{tabular}{|l|l|l|l|l|l|}
\hline $\mathrm{Nt}$ & $\mathrm{Nb}$ & & $\theta^{\prime}(0)$ & & $\beta^{\prime}(0)$ \\
\hline & & Santoshi Misra [1] & & $\begin{array}{l}\text { Santoshi Misra } \\
{[1]}\end{array}$ & \\
\hline 0.1 & 0.1 & 0.08225153 & 0.0825642 & 2.958882 & 2.95905 \\
\hline 0.5 & 0.1 & 0.07928398 & 0.0793975 & 3.000859 & 3.00175 \\
\hline 0.1 & 0.5 & 0.08198826 & 0.0818746 & 2.760671 & 2.761743 \\
\hline 0.5 & 0.5 & 0.07893625 & 0.0785429 & 2.967534 & 2.968632 \\
\hline
\end{tabular}

In every one of these estimations, we have considered $\operatorname{Pr}=2, M=2, R=2, N b=N t=S=A=E c=\gamma=0.5, B i=0.1$ and $L e=5$ 
Table III

\begin{tabular}{|c|c|c|c|c|c|c|c|c|c|c|c|c|c|c|c|}
\hline M & $\mathrm{K}$ & $\beta$ & $\mathrm{R}$ & $\operatorname{Pr}$ & A & $\mathrm{S}$ & $\mathrm{Nt}$ & $\mathrm{Nb}$ & $\mathrm{Ec}$ & Le & $\mathrm{Kr}$ & $\mathrm{Bi}$ & SF & $\mathrm{Nu}$ & Sh \\
\hline 1 & 0.5 & 4 & 2 & 0.71 & 0.5 & 0.5 & 0.5 & 0.5 & 0.5 & 1 & 0.5 & 0.1 & 0.971411 & 0.073878 & 1.076286 \\
\hline 2 & & & & & & & & & & & & & 1.025638 & 0.073949 & 1.083126 \\
\hline 3 & & & & & & & & & & & & & 1.069877 & 0.074059 & 1.091738 \\
\hline 4 & & & & & & & & & & & & & 1.107090 & 0.074230 & 1.102990 \\
\hline 1 & 1 & 4 & 2 & 0.71 & 0.5 & 0.5 & 0.5 & 0.5 & 0.5 & 1 & 0.5 & 0.1 & 0.833794 & 0.074506 & 1.118471 \\
\hline & 2 & & & & & & & & & & & & 0.842294 & 0.074707 & 1.128699 \\
\hline & 3 & & & & & & & & & & & & 0.858521 & 0.074787 & 1.132622 \\
\hline & 4 & & & & & & & & & & & & 0.901927 & 0.074830 & 1.134698 \\
\hline \multirow[t]{4}{*}{1} & 0.5 & 1 & 2 & 0.71 & 0.5 & 0.5 & 0.5 & 0.5 & 0.5 & 1 & 0.5 & 0.1 & 0.847011 & 0.074230 & 1.102990 \\
\hline & & 2 & & & & & & & & & & & 0.922434 & 0.074294 & 1.106765 \\
\hline & & 3 & & & & & & & & & & & 0.953987 & 0.074419 & 1.113794 \\
\hline & & 4 & & & & & & & & & & & 0.971411 & 0.074770 & 1.131551 \\
\hline \multirow[t]{4}{*}{2} & 0.5 & 0.5 & 0.5 & 2 & 0.5 & 0.5 & 0.5 & 0.5 & 0.5 & 1 & 0.5 & 0.1 & 0.796856 & 0.065278 & 1.122883 \\
\hline & & & 1 & & & & & & & & & & 0.796856 & 0.066455 & 1.129657 \\
\hline & & & 1.5 & & & & & & & & & & 0.796856 & 0.067480 & 1.132976 \\
\hline & & & 2 & & & & & & & & & & 0.796856 & 0.067919 & 1.134849 \\
\hline \multirow[t]{4}{*}{2} & 0.5 & 0.5 & 0.5 & 0.71 & 0.5 & 0.5 & 0.5 & 0.5 & 0.5 & 1 & 0.5 & 0.1 & 0.796857 & 0.063986 & 1.106760 \\
\hline & & & & 1 & & & & & & & & & 0.796857 & 0.064668 & 1.119256 \\
\hline & & & & 5 & & & & & & & & & 0.796857 & 0.066400 & 1.138047 \\
\hline & & & & 7 & & & & & & & & & 0.796857 & 0.067799 & 1.139107 \\
\hline \multirow[t]{4}{*}{2} & 0.5 & 0.5 & 0.5 & 0.71 & 1 & 0.5 & 0.5 & 0.5 & 0.5 & 1 & 0.5 & 0.1 & 0.208734 & 0.066064 & 1.046881 \\
\hline & & & & & 2 & & & & & & & & 0.264058 & 0.067091 & 1.056889 \\
\hline & & & & & 3 & & & & & & & & 0.359612 & 0.067273 & 1.073478 \\
\hline & & & & & 4 & & & & & & & & 0.565590 & 0.067285 & 1.106400 \\
\hline
\end{tabular}




\begin{tabular}{|c|c|c|c|c|c|c|c|c|c|c|c|c|c|c|c|}
\hline \multirow[t]{4}{*}{2} & 0.5 & 0.5 & 0.5 & 0.71 & 0.5 & 1 & 0.5 & 0.5 & 0.5 & 1 & 0.5 & 0.1 & 0.828428 & 0.069129 & 1.519874 \\
\hline & & & & & & 2 & & & & & & & 0.891877 & 0.076821 & 2.382039 \\
\hline & & & & & & 3 & & & & & & & 0.954451 & 0.081949 & 3.308736 \\
\hline & & & & & & 4 & & & & & & & 1.014830 & 0.085428 & 4.263773 \\
\hline \multirow[t]{4}{*}{2} & 0.5 & 0.5 & 0.5 & 0.71 & 0.5 & 0.5 & 1 & 0.5 & 0.5 & 1 & 0.5 & 0.1 & 0.796857 & 0.060576 & 1.065693 \\
\hline & & & & & & & 2 & & & & & & 0.796857 & 0.061608 & 1.096135 \\
\hline & & & & & & & 3 & & & & & & 0.796857 & 0.062593 & 1.118847 \\
\hline & & & & & & & 4 & & & & & & 0.796857 & 0.063533 & 1.134140 \\
\hline \multirow[t]{4}{*}{2} & 0.5 & 0.5 & 0.5 & 0.71 & 0.5 & 0.5 & 0.5 & 1 & 0.5 & 1 & 0.5 & 0.1 & 0.796857 & 0.049404 & 1.137881 \\
\hline & & & & & & & & 2 & & & & & 0.796857 & 0.053825 & 1.137998 \\
\hline & & & & & & & & 3 & & & & & 0.796857 & 0.058065 & 1.138176 \\
\hline & & & & & & & & 4 & & & & & 0.796857 & 0.062077 & 1.138539 \\
\hline \multirow[t]{4}{*}{2} & 0.5 & 0.5 & 0.5 & 0.71 & 0.5 & 0.5 & 0.5 & 0.5 & 0.5 & 0.1 & 0.5 & 0.1 & 0.796857 & 0.062700 & 1.135839 \\
\hline & & & & & & & & & & 0.2 & & & 0.796857 & 0.065270 & 1.142372 \\
\hline & & & & & & & & & & 0.4 & & & 0.796857 & 0.067836 & 1.148897 \\
\hline & & & & & & & & & & 0.6 & & & 0.796857 & 0.069117 & 1.152157 \\
\hline \multirow[t]{4}{*}{2} & 0.5 & 0.5 & 0.5 & 0.71 & 0.5 & 0.5 & 0.5 & 0.5 & 0.5 & 1 & 0.5 & 0.1 & 0.796857 & 0.063872 & 1.139107 \\
\hline & & & & & & & & & & 2 & & & 0.796857 & 0.063876 & 1.849592 \\
\hline & & & & & & & & & & 3 & & & 0.796857 & 0.063896 & 2.490057 \\
\hline & & & & & & & & & & 4 & & & 0.796857 & 0.063986 & 3.095380 \\
\hline \multirow[t]{4}{*}{2} & 0.5 & 0.5 & 0.5 & 0.71 & 0.5 & 0.5 & 0.5 & 0.5 & 0.5 & 1 & 1 & 0.1 & 0.796857 & 0.063892 & 1.383936 \\
\hline & & & & & & & & & & & 2 & & 0.796857 & 0.063900 & 1.759857 \\
\hline & & & & & & & & & & & 3 & & 0.796857 & 0.063916 & 2.059805 \\
\hline & & & & & & & & & & & 4 & & 0.796857 & 0.063949 & 2.316880 \\
\hline \multirow[t]{3}{*}{2} & 0.5 & 0.5 & 0.5 & 0.71 & 0.5 & 0.5 & 0.5 & 0.5 & 0.5 & 1 & 0.5 & 0.2 & 0.796857 & 0.098292 & 1.144968 \\
\hline & & & & & & & & & & & & 0.4 & 0.796857 & 0.133942 & 1.150758 \\
\hline & & & & & & & & & & & & 0.6 & 0.796857 & 0.152201 & 1.153602 \\
\hline
\end{tabular}




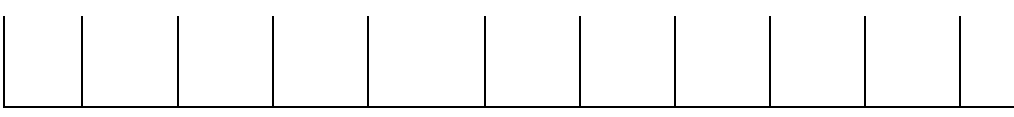

Conclusions:

From the above discussion, we can make the following conclusions.

1. The velocity decreases with the increased parametric values of magnetic (M), Casson fluid $(\beta)$, slip (A) and suction (S), but the reverse trend is observed in permeability parameter $(\mathrm{K})$.

2. Temperature increases with the rises of radiation parameter $(\mathrm{R})$, thermophoresis parameter $(\mathrm{Nt})$, Brownian motion parameter $(\mathrm{Nb})$, Eckert number (Ec) andBiot number $(\mathrm{Bi})$ whereas the reverse process detected in the prandtl number (Pr).

3. The concentration profiles enhance for the growing values of Brownian motion parameter $(\mathrm{Nb})$ and decrease for the increased values of thermophoresis $(\mathrm{Nt})$, Lewis number (Le) and chemical reaction (Kr) parameters.

4. Nusselt and Sherwood numbers raises with the enhance of $\mathrm{M}, \mathrm{K}, \beta, \mathrm{R}, \mathrm{Pr}, \mathrm{A}, \mathrm{S}, \mathrm{Nt}$, $\mathrm{Nb}, \mathrm{Ec}$, Le and Bi respectively

5. Skin friction coefficient rises with the parameters $\mathrm{M}, \mathrm{K}, \beta$, A and $\mathrm{S}$. But being constant with the parameters $\mathrm{R}, \mathrm{Pr}, \mathrm{Nt}, \mathrm{Nb}$, Ec, Le, Kr, Bi.

\section{References}

[1] Santoshi, M., Narender, G. and Govardha, K., (2019). Numerical solution of boundary layer flow of MHD nanofluid over a stretching surface with chemical reaction and viscous dissipation effects. Open journal of mathematical sciences, 3, 289-299.

[2] Tripathy, R. S., Dash, G. C., Mishra, S. R. and Baag, S., (2015). Chemical reaction effect on MHD free convective surface over a moving vertical plate through \begin{tabular}{l|l|l|}
1 & 0.796857 & 0.170705
\end{tabular}

1.156399

porous medium. Alexandria Engineering Journal, 54(3), 673-679.

[3] Fang,T.,Chia-fon, F.L. and Zhang,J., (2011).

Theboundarylayersofanunsteadyincompres siblestagnation-pointflow with mass transfer. International Journal of NonLinear Mechanics, 46(7), 942-948.

[4] Binyam Zigta., (2019). Thermal radiation, chemical reaction and viscous dissipation effects on unsteady MHD flow of viscoelastic fluid embedded in a porous medium. Budapest International Research in Exact Sciences Journal, 1(3), 35-57.

[5] Crane, L.J., (1970). Flow past a stretching plate. Journal of Applied Mathematics and Physics (ZAMP), 21(4), 645-647.

[6] Ramana Reddy, G. V. and Hari Krishna, Y., (2018). MHD free convective flow of Casson fluid past over an oscillating vertical porous plate. International Journal of Mathematical Archive, 9(5), 119-126.

[7] Ramana Reddy, G. V., Jayarami Reddy, K. and Lakshmi, R., (2015). Radiation and Mass transfer Effects on nonlinear MHD boundary layer flow of liquid metal over a porous stretching surface embedded in porous medium with heat generation. WSEAS Transactions on Fluid Mechanics, 10, 1-12.

[8] Raja Sekhar, K., Ramana Reddy, G. V. and Prasad, B. D. C. N., (2012). Chemically Reacting on MHD Oscillatory Slip Flow in a Planer Channel with Varying Temperature and Concentration. Advances in Applied Science Research, 3 (5), 2652-2659.

[9] Chiam, T. C. (1995). Hydromagnetic flow over a surface stretching with a power-law velocity. International Journal of Engineering Science, 33(3), 429-435.

[10] Rahman Elbashbeshy, E.M.A., Mohamed Abdelgaber, K. and Asker, 
H.G.,(2018). Heat and mass transfer of a Maxwell nanofluid over a stretching surface with variable thickness embedded in porous medium. International Journal of Mathematics and Computational Science, 4(3), 86-98.

[11] Suresh, P., Hari Krishna,Y.,Sreedhar Rao,R. and Janardhana Reddy, P. V., (2019). Effect of Chemical Reaction and Radiation on MHD Flow along a moving Vertical Porous Plate with Heat Source and Suction.International Journal of Applied Engineering Research, 14(4), 869-876.

[12] Shi jun liao, Ioan pop, (2004). Explicit analytic solution for similarity boundary layer equations. International journal of heat and mass transfer, 47,7585.

[13] Reddy, M.G., (2014). Influence of MHD and thermal radiation boundary layer flow of a nanofluid past a stretching sheet. Journal of scientific research, 6 (2), 257-272.

[14] Shateyi, S., Mabood2,F.and Lorenzini, G., (2017). Casson fluid flow free convective heat and mass transfer over an unsteady permeable stretching surface considering viscous dissipation. Journalof engineering thermophysics, 26, 39-52.

[15] Chamkha, A. J.and Issa, C. (2000). Effects of heat generation/absorption and thermophoresis on hydromagnetic flow with heat and mass transfer over a flat surface. International Journal of Numerical Methods for Heat \& Fluid Flow, 10(4), 432-449.

[16] Gebhart, B. and Mollendorf,J.(1969).

Viscousdissipationinexternalnaturalconvec tionflows. Journal of fluid Mechanics, 38(1), 97-107.

[17] Vajravelu, K. (2001). Viscous flow over a nonlinearly stretching sheet.
Applied mathematics and computation, 124(3), 281-288.

[18] Cortell, R. (2005). Flow and heat transfer of a fluid through a porous medium over a stretching surface with internal heat generation/absorption and suction/blowing. Fluid Dynamics Research, 37(4), 231.

[19] Khan, S. K., and Sanjayanand, E. (2005). Viscoelastic boundary layer flow and heat transfer over an exponential stretching sheet. International Journal of Heat and Mass Transfer, 48(8), 15341542.

[20] Akyildiz, F. T., Bellout, H. and Vajravelu, K. (2006). Diffusion of chemically reactive species in a porous medium over a stretching sheet. Journal of Mathematical Analysis and Applications, 320(1), 322-339.

[21] Khan, W. A. and Pop, I. (2011). Boundary-layer flow of a nanofluid past a stretching sheet. International Journal of Heat and Mass Transfer, 53, 2477-2483.

[22] Makinde, O. D. and Aziz, A. (2011). Boundary layer flow of a nanofluid past a stretching sheet with a convective boundary condition. International Journal of Thermal Sciences. 50, 1326-1332

[23] of Thermal Sciences. 50, 13261332 